\title{
SYNCHRONOUS PRESENTATION OF INVASIVE DUCTAL BREAST CARCINOMA AND FOLLICULAR LYMPHOMA: A DIAGNOSTIC CHALLENGE
}

Priscila Nunes Silva Morosini', Murilo do Vale Sabóia ${ }^{1}$, Teresa Cristina Santos Cavalcanti² ${ }^{2}$ Ágata Rothert ${ }^{1}$, Marcela Santos Cavalcanti²

${ }^{1}$ Hospital São Vicente - Curitiba (PR), Brazil.

${ }^{2}$ Neopath Patologia Diagnóstica - Curitiba (PR), Brazil.

Introduction: The presentation of synchronous tumors is rare. At the same time, the increase in the incidence of non-Hodgkin lymphoma in patients treated for malignant breast neoplasm submitted to radiotherapy is a known fact. However, some authors have reported cases of breast neoplasm and lymphoma at initial diagnosis. It is unclear whether they originate from common underlying mechanisms, triggering others, or if one disease process is completely independent of the other. Clinical case: A 69-year-old asymptomatic female patient was referred to the mastology department due to abnormalities in the routine mammography. Upon presentation, she had no associated B symptoms, and the physical examination revealed a palpable nodule in the left breast and suspicious palpable left axillary lymphadenopathy. Mammography prior to the appointment showed a $15 \mathrm{~mm}$ nodule in the left breast with well-defined margins. A complementary ultrasound revealed multiple simple cysts in the left breast, the largest with $1.3 \mathrm{~cm}$ and retroareolar. The anatomopathological report of the core biopsy and fine-needle aspiration biopsy (FNAB) indicated an invasive ductal carcinoma in the left breast, with T2N2M0 as the initial clinical staging. Immunohistochemical evaluation revealed estrogen receptor-positive (ER+++ 95\%), progesterone receptor-negative (PR-), HER2-, Ki67 8\%. FNAB of axillary lymph node showed no malignancy in the sample. Staging tomography had no evidence of distant lesions. The patient underwent a radical mastectomy and axillary lymph node dissection with plans for adjuvant chemotherapy. The final anatomopathological report of the surgical specimen revealed a well-differentiated invasive ductal breast carcinoma associated with intraductal carcinoma, measuring $2.7 \times 1.9 \times 1.8 \mathrm{~cm}$ and with free margins. A total of 45 lymph nodes were dissected, with no evidence of involvement by carcinoma. However, an atypical proliferation strongly suggestive of follicular lymphoma was identified. Immunohistochemistry was positive for CD 10, Bcl-6, and Bcl-2, compatible with follicular lymphoma, grade 1-2 (predominantly follicular $>75 \%$ ). Discussion: Literature reviews show that $88.9 \%$ of case reports have failed in diagnosing the second synchronous neoplasm. Usually, FNAB and even core biopsy of these lymph nodes does not guarantee the diagnosis, given the high rates of false-negative in these cases, and their findings are often insufficient. Imaging diagnosis is frequently unclear in these situations, and the diagnosis is mainly reached after surgical treatment and final histological evaluation. Final considerations: The case brings to light the discussion about the treatment of a complex, hard to diagnose situation, which leads to delayed management. Multidisciplinary follow-up is crucial for this diagnosis so as to prevent unfavorable outcomes. 